\title{
DESEMPENHO DE TRÊS TEORIAS DE SIMULAÇÃO DO COMPORTAMENTO DE UM TRATOR AGRÍCOLA EM TRAÇÃ ${ }^{1}$
}

\author{
JOSÉ F. SCHLOSSER ${ }^{2}$, PILAR LINARES ${ }^{3}$, EDUARDO G. DE SOUSA FILHO ${ }^{4}$
}

\begin{abstract}
RESUMO: A simulação matemática é uma ferramenta importante na diminuição de custos de produção e viabilização de novos projetos de máquinas agrícolas. Este trabalho trata da comparação entre três teorias de simulação, as chamadas teorias de predição de tração clássicas, desenvolvidas por Wismer, Brixius e Gee-Clough, na simulação do desempenho de um trator agrícola de rodas. Para efetuar uma comparação entre as três teorias e apurar as condições mais apropriadas de uso das mesmas, realizou-se um experimento de campo com um trator de tração simples (4x2), com o controle de um número apreciável de variáveis relevantes, para criar condições de comparação com uma simulação posterior. No trabalho de campo, foram utilizados tratamentos que envolviam a condição do solo, preparado ou consolidado; pneus do tipo diagonal, e a deflexão desse pneu, maior e menor. Utilizando os parâmetros medidos a campo, realizou-se a comparação com os dados simulados para a variável patinamento. A teoria $\mathrm{Cn}$ mostrou que possui uma faixa de utilização mais restrita do que as teorias $\mathrm{Bn}$ e $\mathrm{MN}$, sendo o índice de cone a variável que determina essas condições de uso.
\end{abstract}

PALAVRAS-CHAVE: teorias de predição, patinamento, índice de cone.

\section{EVALUATION OF THREE THEORIES FOR PREDICTING THE PERFORMANCE OF AGRICULTURAL TRACTORS}

SUMMARY: The simulation of machines behavior by mathematical modeling is nowadays an important tool for reducing production costs and making new projects feasible. The present study comprises a comparison among three theories for predicting the traction performance of a tractor, developed by Wismer, Brixius and Gee-Clough. In order to compare the three theories and find out the most appropriate conditions for using them, a field experiment in which all relevant variables were controlled for further simulation was conducted by using a $2 \mathrm{WD}$ tractor $(4 \times 2)$. Using the parameter obtained in field, a comparison was carried out with the results from the different simulation models. In this field study was used treatments that involved soil conditions, with grass and conventional tillage; diagonal tire; and the deflection of this tire, one bigger and the other smaller. Based on those, a comparative analysis of slipping was performed between the measured and the simulated data. $\mathrm{Cn}$ theory showed to have lower precision when compared to $\mathrm{Bn}$ and $\mathrm{MN}$ theories being the cone index the variable which determines such conditions.

KEYWORDS: traction prediction, slipping, cone index.

\footnotetext{
${ }^{1}$ Extraído da dissertação de mestrado do terceiro autor.

${ }^{2}$ Eng ${ }^{\mathrm{o}}$ Agrônomo, Prof. Titular, Departamento de Engenharia Rural, UFSM, Santa Maria - RS, Fone: (0XX55) 220.8175, e-mail: schlosse@ccr.ufsm.br

${ }^{3}$ Eng $^{\text {o }}$ Agrônomo, Prof. Titular, Departamento de Ingeniería Rural, ETSIA, UPM, Madrid, Espanha.

${ }^{4}$ Eng ${ }^{0}$ Agrônomo, M.Sc., AGCO, Canoas - RS.

Recebido pelo Conselho Editorial em: 13-5-2003

Aprovado pelo Conselho Editorial em: 26-7-2004
} 


\section{INTRODUÇÃO}

O estudo do desempenho de tratores agrícolas no campo é uma tarefa bastante complexa, pois a quantidade de variáveis a serem controladas e analisadas é muito grande. O conjunto mecânico possui diversos pontos que devem ser monitorados, seja por instrumentação e/ou metodologias específicas de controle, o que pode proporcionar subsídios para analisar seu desempenho. Esse monitoramento pode ser difícil e absorver bastante trabalho. Porém, quando se inclui o terreno onde está desenvolvendo tração, percebe-se que fica praticamente inexeqüível controlar todas as variáveis pela quantidade e dificuldade de medi-las. Em diversas situações, estudou-se uma forma prática de determinar uma eficiente maneira de diferenciar a qualidade de tração dos tratores e veículos extraviários. Dessa forma, surgiram algumas teorias de simulação, que ,em comum, têm por objetivo estimar o comportamento de vários parâmetros de rendimento desses veículos.

As equações utilizadas baseiam-se em diversas variáveis que podem ser medidas direta e indiretamente e que caracterizam de maneira conjunta o solo e a máquina. Assim, cada uma das partes desse sistema pode ser demonstrada por variáveis específicas.

O solo representa o meio sobre e no qual o veículo vai deslocar-se e pode ser caracterizado pelo índice de cone. O veículo é o elemento que, por seu peso e distribuição desse sobre os eixos, faz com que a aplicação das pressões ao solo varie conforme suas dimensões características e condições de trabalho. Os pneus são caracterizados em suas dimensões e as alterações que essas sofrem com a variação das cargas aplicadas.

As teorias de predição clássicas são $C n, B n$ e $M N$, conforme reconhecem vários autores que trabalham no tema. Na teoria $C n$, de WISMER \& LUTH (1972), a relação entre solo e veículo é determinada por um parâmetro denominado Número Característico da Roda $(\mathrm{Cn})$, o qual congrega valores dos três elementos principais: solo, com índice de cone; veículo, com seu peso dinâmico, e o pneu, por suas medidas características. Baseado nesse parâmetro, realiza-se a simulação. Na teoria $B n$, de BRIXIUS (1975), e na teoria $M N$, de GEE-CLOUGH et al. (1978), os autores basearam-se na teoria anterior e em ensaios realizados pela Companhia Deere e em outros centros de investigação para aprimorar os modelos, melhorando a precisão da simulação.

Os modelos matemáticos utilizados na predição proporcionam economia de tempo e grande redução nos custos dos projetos. Como exemplo, tem-se MÁRQUEZ (1980), que utilizou as teorias de predição para prever, em pista de concreto, o comportamento de um trator aproveitando dados dos ensaios de motor em dinamômetro. Mas as teorias básicas têm aplicabilidade para a média das situações ou para uma determinada condição local, o que gera necessidade de adaptação dessas teorias para as condições de outros meios e situações.

SERPA (1997) desenvolveu um modelo matemático para a simulação do desempenho de tratores em trabalhos de preparo do solo em que essa foi realizada pela teoria $C n$, a qual avaliou bem o comportamento dos mesmos em uma faixa de patinamento entre 8 e $18 \%$.

Este trabalho teve como objetivo principal determinar quais são as condições mais adequadas de utilização das três diferentes teorias de predição do comportamento do trator agrícola testadas, bem como avaliar a que tem melhor desempenho na simulação.

\section{MATERIAL E MÉTODOS}

O trabalho foi conduzido em duas fases distintas. Na primeira, realizou-se um experimento de campo com condições preestabelecidas, quando foram medidas as diferentes variáveis de comportamento do trator. Na segunda, realizou-se a simulação de comportamento do trator com base nas mesmas condições do trabalho de campo, por meio das três diferentes teorias de predição: $C n, B n$ e $M N$. 
O experimento de campo foi conduzido sob duas condições de solo, preparado e não preparado. Havia duas áreas de igual dimensão, sendo uma com o solo preparado e outra de pastagem nativa roçada, caracterizando as condições de solo preparado e consolidado, respectivamente. $\mathrm{O}$ solo onde se realizaram os trabalhos é classificado como Podzólico Vermelho-Amarelo distrófico, unidade de mapeamento São Pedro, bem drenado, com relevo plano a levemente ondulado.

O índice de cone foi medido para cada repetição de cada tratamento, sendo o componente representativo do solo na simulação matemática do comportamento do trator. O equipamento utilizado foi um penetrógrafo gráfico da marca Soilcontrol, modelo SC-60, com dimensões, funcionamento e procedimentos de utilização em acordo com a norma ASAE S313.2. Após a tomada de dados a campo, os gráficos gerados pelo penetrógrafo foram analisados, resultando os valores dos índices de cone. A umidade gravimétrica foi obtida conforme EMBRAPA (1997). O índice de cone foi medido antes da passada do trator, exatamente sobre a área onde os pneus passariam exercendo a tração. Esses pontos eram locados aleatoriamente sobre a projeção das rodas na parcela.

Os tratamentos, no campo, foram compostos por dois fatores, com dois níveis e três repetições cada um (Tabela 1). Com base nesta composição de tratamentos, efetuou-se a análise dos dados de simulação em relação aos de campo.

TABELA 1. Tratamentos usados no experimento.

\begin{tabular}{ccccc}
\hline Tratamento & Solo & Pneu & Deflexão & Repetição \\
\hline CDME & Consolidado & Diagonal & Menor & 1,2 e 3 \\
CDMA & Consolidado & Diagonal & Maior & 1,2 e 3 \\
PDME & Preparado & Diagonal & Menor & 1,2 e 3 \\
PDMA & Preparado & Diagonal & Maior & 1,2 e 3 \\
\hline
\end{tabular}

O trator-teste era posicionado à frente, unido ao trator-freio por um cabo de aço, no qual estava acoplada uma célula de carga para medir o esforço de tração. $O$ trator-teste possuía uma plataforma de instrumentação acoplada aos braços do sistema hidráulico que recebia sinais da célula de carga. Ambos os tratores com o diferencial bloqueado faziam um percurso de $60 \mathrm{~m}$, sendo os $10 \mathrm{~m}$ iniciais e finais desconsiderados nas medições de esforços de tração e patinamento.

Como trator-teste, foi utilizado o modelo MF 275 da marca Massey Ferguson, com potência bruta no motor de $56,6 \mathrm{~kW}(77 \mathrm{cv})$ a $2.000 \mathrm{rpm}$, cilindrada de $3.865 \mathrm{~cm}^{3}$ e com tração simples. A massa total desse trator era de $3.080 \mathrm{~kg}$, sem lastro metálico dianteiro ou traseiro e com a adição de lastro líquido nas rodas motrizes traseiras. A distribuição de massas do trator foi de $34 \%$ no eixo dianteiro e $66 \%$ no eixo traseiro. Durante as avaliações, o trator-teste foi utilizado com uma rotação no motor de $1.900 \mathrm{rpm}$, em todos os tratamentos, com uma velocidade teórica de $5,5 \mathrm{~km} \mathrm{~h}^{-1}$. Em todos os tratamentos, o trator permaneceu com o bloqueio do diferencial acionado.

O trator-freio utilizado foi da marca Massey Ferguson, modelo 5310 4x2 TDA, com massa de $4.000 \mathrm{~kg}$. Para a seleção das cargas que impuseram diferentes patinamentos ao trator-teste, selecionaram-se marchas e rotações do motor do trator-freio por meio de testes prévios, visando a provocar carga ao trator-teste, atingindo determinado nível de patinamento. Esses testes eram realizados na área do experimento, imediatamente antes da realização das provas. No trator-freio, selecionavam-se a marcha e a rotação do motor e, com o diferencial bloqueado, iniciava-se o teste, medindo-se o patinamento do trator, determinando três níveis de patinamento distintos. Os três níveis correspondiam a três cargas com diferentes exigências: uma de nível baixo, uma intermediária e outra de nível alto, com valores de patinamento em torno de $10 \% ; 15 \%$ e $20 \%$, respectivamente. Na Tabela 2 , descrevem-se as marchas selecionadas e os níveis de carga atingidos. 
TABELA 2. Seleção das marchas e rotações do motor do trator-freio, para três cargas nos tratamentos.

\begin{tabular}{cccc}
\hline Tratamento & Carga Alta & Carga Média & Carga Baixa \\
\hline PDME e PDMA & $5^{\mathrm{a}} 800 \mathrm{rpm}$ & $5^{\mathrm{a}} 1.600 \mathrm{rpm}$ & $6^{\mathrm{a}} 1.300 \mathrm{rpm}$ \\
CDME e CDMA & $5^{\mathrm{a}} 1.100 \mathrm{rpm}$ & $5^{\mathrm{a}} 1.500 \mathrm{rpm}$ & $6^{\mathrm{a}} 1.100 \mathrm{rpm}$ \\
\hline
\end{tabular}

O pneu utilizado, de carcaça diagonal, foi da marca Goodyear, modelo Super Arrozeiro, de fabricação nacional, com a designação 23.1-26 (R2) de 8 lonas.

A deflexão dos pneus varia conforme o peso que gravita sobre a roda e a pressão interna do pneu. A maneira como se realizaram as medidas estáticas, estava de acordo com a norma ASAE S296.3 (1995).

TABELA 3. Deflexões dos pneus usados nos testes de campo.

\begin{tabular}{lccc}
\hline Pneu & Pressão interna & \multicolumn{2}{c}{ Deflexão } \\
\hline Diagonal & $3,19 \mathrm{kPa}$ & Menor & $9,5 \%$ \\
Diagonal & $1,45 \mathrm{kPa}$ & Maior & $17,5 \%$ \\
\hline
\end{tabular}

Como instrumento de medida do esforço de tração do trator-teste, foi utilizada uma célula de carga marca Alfa Instrumentos, colocada na barra de tração, com capacidade para medir esforços de até $50 \mathrm{kN}$, e um condicionador de sinais com visor de cristal líquido, o qual mostra os valores de carga instantânea. O funcionamento é baseado em pulsos elétricos que, gerados na célula de carga, são transformados no condicionador de sinais em números que são exibidos no visor. $\mathrm{O}$ peso de toda a instrumentação, com sua plataforma, é de $2,2 \mathrm{kN}$. Uma bateria de $12 \mathrm{~V}$ supriu as necessidades de energia da instrumentação.

Para a medição do patinamento, utilizou-se o método das balizas, descrito em LINARES (1996), o qual usa balizas e trena na medição do deslocamento do trator para um determinado número de voltas da roda. O patinamento das rodas motrizes foi medido contando cinco voltas da roda e, conseqüentemente, a distância percorrida. Com esse dado, efetuava-se a comparação com condição de referência.

Para a realização das simulações do comportamento dos tratores em trabalho, utilizando modelos matemáticos, necessitam-se dados do solo agrícola onde os tratores desenvolvem tração e dados ponderais e dimensionais do trator que está sendo testado. Devem ser determinadas as dimensões dos pneus que o equipam, pois esses são dados de entrada nas diferentes predições.

O solo agrícola, meio onde ocorre o trabalho dos tratores, é caracterizado para todas as teorias por meio do índice de cone obtido por um penetrógrafo. Como esse instrumento expressa pressão de um cone sobre o solo, a unidade utilizada é o $\mathrm{kPa}$.

A caracterização dos pneus nas simulações é feita pelas dimensões. Na simulação, utilizando os modelos matemáticos, as entradas dessas variáveis são o metro, para as dimensões dos pneus; o bar, para a pressão interna, e o kgf, para a carga vertical sobre o rodado. Em determinados casos, é justificável o uso desses dados diretamente das informações técnicas fornecidas pelos fabricantes, pois a proposta da predição é evitar a necessidade de ir ao campo para realizá-la, no máximo medindo os índices de cone do solo objeto do estudo.

Do trator, é necessário informar o peso estático, a distribuição desse sobre os eixos e, por consequiência, sobre as rodas. É necessário informar dados dimensionais do trator, como a altura do ponto de engate e a distância entre eixos para que o modelo de simulação calcule a condição dinâmica de transferência de peso e a carga na barra de tração do trator-teste. 
Para a comparação entre o previsto nas teorias e o medido no campo, foram aplicados aos modelos os valores medidos no experimento. As simulações foram feitas conforme preconizam os trabalhos de WISMER \& LUTH (1972) para teoria $C n$; BRIXIUS (1975) para a teoria $B n$ e GEECLOUGH et al. (1978) para a teoria $M N$.

\section{RESULTADOS E DISCUSSÃO}

O solo da área do experimento foi amostrado e determinada sua composição granulométrica, apresentando $41,7 \%$ de silte $(0,05$ a $0,002 \mathrm{~mm}), 28,6 \%$ de areia fina $(0,2$ a $0,05 \mathrm{~mm})$, podendo ser classificado como de classe textural franca.

A comparação entre dados de patinamento calculados e medidos no campo foi feita com base em teste estatístico chamado de Teste $t$.

$\mathrm{Na}$ análise para o tratamento CDME, a teoria $C n$, conforme o Teste $t$ (Tabela 4), obteve, para esse tratamento, resultados que diferiram significativamente dos dados de campo para todos os níveis de patinamento, observando-se tendência de subestimação, fato que se repetiu no tratamento CDMA para essa teoria, conforme Tabela 5. Para a teoria $B n$, no tratamento CDME, nos patinamentos baixos e médios, os valores simulados não diferiram significativamente dos medidos no ensaio de campo; porém, no patinamento mais alto, os resultados diferiram significativamente, com tendência de subestimar os valores. Já para o tratamento CDMA, os patinamentos calculados não diferiram significativamente em todos os níveis avaliados. A teoria $M N$ apresentou valores que não diferiram significativamente dos obtidos no ensaio, para todos os níveis de patinamentos estipulados no tratamento CDME; já o tratamento CDMA apresentou diferenças significativas no nível alto de patinamento, enquanto, nos demais, os dados calculados corresponderam aos medidos, conforme a Tabela 5 .

TABELA 4. Resumo do Teste $t$ para o tratamento CDME entre os dados medidos e simulados com seus índices de cone.

\begin{tabular}{lccccc}
\hline \multicolumn{4}{c}{ Resultados do Teste $t$} & & $\begin{array}{c}\text { IC Médio do Tratamento } \\
(\mathrm{kPa})\end{array}$ \\
\cline { 1 - 4 } Tratamento & DC $\mathrm{x} C$ & $\mathrm{DC} \times \mathrm{Bn}$ & $\mathrm{DC} \times M N$ & \\
\hline CDME - baixo & DS & NS & NS & 1.357 \\
CDME - médio & DS & NS & NS & 1.357 \\
CDME - alto & DS & DS & NS & 1.357
\end{tabular}

DS - diferença significativa entre os valores de campo e simulados; NS - não há diferença significativa entre os valores de campo e simulados; $\mathrm{S}(\mathrm{p}<0,05)$.

TABELA 5. Resumo do Teste $t$ para o tratamento CDMA entre os dados medidos e simulados com seus índices de cone.

\begin{tabular}{lcccc}
\hline \multicolumn{3}{c}{ Resultados do Teste $t$} & & $\begin{array}{c}\text { IC Médio do Tratamento } \\
(\mathrm{kPa})\end{array}$ \\
\cline { 1 - 4 } Tratamento & DC $\mathrm{x} n$ & $\mathrm{DC} \times \mathrm{B} n$ & $\mathrm{DC} \times M N$ & \\
\hline CDMA - baixo & DS & NS & NS & 1.313 \\
CDMA - médio & DS & NS & NS & 1.313 \\
CDMA - alto & DS & NS & DS & 1.313
\end{tabular}

DS - diferença significativa entre os valores de campo e simulados; NS - não há diferença significativa entre os valores de campo e simulados; $\mathrm{S}(\mathrm{p}<0,05)$.

Para os tratamentos CDME e CDMA, percebe-se que a teoria $C n$ não obteve bons resultados de simulação. Esse desempenho confirma a sensibilidade de tal simulação aos valores de índices de cone, efeito já citado por CERVANTES (1993) e HERNANDEZ (1999). Já as teorias Bn e $M N$, mostraram- 
se menos sensíveis ao "efeito solo" que a anterior, apresentando resultados que, na maioria das condições, não diferiram significativamente, com tendência de superestimação dos valores nos patinamentos mais altos.

O tratamento PDME e o tratamento PDMA, para a teoria $C n$, apresentaram resultados de simulação que diferem significativamente dos obtidos no campo, nos patinamentos baixo e médio; já para o nível mais alto de patinamento, em torno de $20 \%$, não houve diferenças significativas, o que discorda dos resultados de SERPA (1997), que obteve melhores resultados de simulação em até $18 \%$ de patinamento. Para as teorias $B n$ e $M N$, os resultados de simulação não diferiram significativamente dos medidos no campo, em todos os níveis de patinamento testados no tratamento PDME. O tratamento PDMA apresentou resultados simulados com diferença significativa nos patinamentos inferiores, com tendência de subestimação; para os demais, os resultados não diferiram significativamente, conforme Tabelas 6 e 7.

TABELA 6. Resumo do Teste $t$ para o tratamento PDME com os índices de cone.

\begin{tabular}{|c|c|c|c|c|}
\hline \multicolumn{4}{|c|}{ Resultados do Teste $t$} & \multirow{2}{*}{ IC Médio do Tratamento $(\mathrm{kPa})$} \\
\hline Tratamento & $\mathrm{DC} \times \mathrm{Cn}$ & $\mathrm{DC} \times B n$ & $\mathrm{DC} \times M N$ & \\
\hline PDME - baixo & DS & NS & NS & 647 \\
\hline PDME - médio & DS & NS & NS & 647 \\
\hline PDME - alto & NS & NS & NS & 647 \\
\hline
\end{tabular}

DS - diferença significativa entre os valores de campo e simulados; NS - não há diferença significativa entre os valores de campo e simulados; $\mathrm{S}(\mathrm{p}<0,05)$.

TABELA 7. Resumo do Teste $t$ para o tratamento PDMA com os índices de cone.

\begin{tabular}{|c|c|c|c|c|}
\hline \multicolumn{4}{|c|}{ Resultados do Teste $t$} & \multirow{2}{*}{ IC Médio do Tratamento $(\mathrm{kPa})$} \\
\hline Tratamento & $\mathrm{DC} \times C n$ & $\mathrm{DC} \times B n$ & $\mathrm{DC} \times M N$ & \\
\hline PDMA - baixo & $\overline{\mathrm{DS}}$ & $\mathrm{DS}$ & DS & 843 \\
\hline PDMA - médio & DS & NS & NS & 843 \\
\hline PDMA - alto & NS & NS & NS & 843 \\
\hline
\end{tabular}

DS - diferença significativa entre os valores de campo e simulados; NS = não há diferença significativa entre os valores de campo e simulados; $\mathrm{S}(\mathrm{p}<0,05)$.

\section{CONCLUSÕES}

A teoria $\mathrm{Cn}$ mostrou-se inadequada para solos consolidados, com índices de cone acima de $1.000 \mathrm{kPa}$.

As teorias $B n$ e $M N$ mostraram-se menos sensíveis à variação do índice de cone que a teoria $C n$, apresentando melhor desempenho que essa para solos consolidados.

As teorias mais apropriadas de desempenho de simulação foram $B n$ e $M N$, enquanto a teoria $C n$ apresentou um desempenho inferior.

Recomenda-se que os trabalhos sobre teorias de predição devem ter continuidade, pois as mesmas se apresentaram como ferramentas bastante úteis na simulação do comportamento do trator agrícola. Os próximos trabalhos sobre o tema devem procurar os valores limites de uso de cada teoria, de forma mais precisa, buscando intervalos de valores de índices de cone mais próximos, bem como optar por trabalhar somente com uma teoria, aprofundando as possibilidades de uso dessa. 


\section{REFERÊNCIAS}

ASAE STANDARD. Soil cone penetrometer. St. Joseph: American Society of Agricultural Engineering, 1988. p. 683. (ASAE Standard: S313.2)

ASAE STANDARD. Uniform terminology for traction of agricultural tractors, self-propelled implements, and other traction and transport device. St. Joseph: American Society of Agricultural Engineering, 1995. p.119-21. (ASAE Standard: S296.3)

BRIXIUS, W.W. Traction prediction equations for wheeled vehicles. Moline: Deere \& Company, 1975. 22 p. (Documento n. 109, Projeto n. 9008: Tecnical Center)

CERVANTES, V.M. Estudio experimental del sistema suelo-vehículo aplicado a la predicción de tracción de los tractores agrícolas. 1993. 143 f. Tese (Doutorado em Energia, Máquinas e Irrigação) Universidade Politécnica de Madrid, Madrid, Espanha, 1993.

GEE-CLOUGH, D.; McALLISTER, M.; EVERNDEN, D.W. Tractive performance of drive tyres. Journal of Agricultural Engineering Research, London, v.22, n.4, p.373-404, 1978.

GOODYEAR. Guia de comprador de pneus agrícolas. [S.L.], 1998. 19 p.

HERNANDEZ, P.M.A. Analisis del patinamiento en tractores de simple traccion atraves de las teorias de prediccion de traccion de ASAE y su contraste experimental. 1999. $140 \mathrm{f}$. Tese (Doutorado em Energia, Máquinas e Irrigação) - Universidad Politécnica de Madrid, Madrid, Espanha, 1999.

LINARES, P. Teoria de la tracción de tractores agrícolas. Madrid: Universidad Politécnica de Madrid, 1996. 157 p.

MÁRQUEZ, L. Predicción del comportamiento de un tractor a la barra a partir del ensayo de su motor en banco. 1980. 420 f. Tese (Doutorado em Energia, Máquinas e Irrigação) - Universidade Politécnica de Madrid, Madrid, Espanha, 1980.

SERPA, F.M. Estimativa de esforços em implementos de preparo de solos e adequação trator implemento. 1997. 94 f. Dissertação (Mestrado em Projeto e Desenvolvimento de Máquinas Agrícolas) - Faculdade de Engenharia Agrícola, Universidade Estadual de Campinas, Campinas, 1997.

WISMER, R.D.; LUTH, H.J. Off-road traction prediction of wheeled vehicles. Transactions of the $A S A E$, St. Joseph, v.72, n.619, p.15, 1972. 